\title{
ON THE COMPACTNESS \\ OF STRONGLY CONTINUOUS SEMIGROUPS AND COSINE FUNCTIONS OF OPERATORS
}

\author{
HERNÁN R. HENRỊQUEZ
}

(Communicated by Palle E. T. Jorgensen)

\begin{abstract}
In this note we relate two notions of compactness for strongly continuous semigroups of linear operators and cosine functions of linear operators. Specifically, if $T$ denotes a strongly continuous semigroup of linear operators defined on a Banach space $X$, we will show that $T$ is compact if and only if the set $\{(T(\cdot) x: x \in X,\|x\| \leq 1\}$ is relatively compact in any space $\left.L^{p}([0, a]) ; X\right)$ for $1 \leq p<\infty$ and $a>0$. We establish similar results for $(T(t)-I)^{n}, n \in \mathbf{N}$, and for cosine and sine functions of operators.
\end{abstract}

\section{INTRODUCTION}

In this note we will present another characterization for the compactness of strongly continuous semigroups and cosine functions of linear operators. For the necessary concepts of semigroup and cosine function of linear operators theory we refer to Nagel [8] and Fattorini [2], respectively.

Throughout this work we will denote by $X$ a Banach space endowed with a norm $\|\cdot\|$ and we will represent by $\mathscr{B}(X)$ the Banach algebra of bounded linear operators defined on $X$.

Let $A$ be the infinitesimal generator of a strongly continuous semigroup $T$ on $X$. A useful condition to obtain existence of mild solutions for an abstract Cauchy problem

$$
\begin{aligned}
\dot{x}(t) & =A x(t)+F(t, x(t)), \quad t \geq 0, \\
x(0) & =x_{0}
\end{aligned}
$$

or for an abstract functional Cauchy problem

$$
\begin{aligned}
& \dot{x}(t)=A x(t)+F\left(t, x_{t}\right), \quad t \geq 0, \\
& x_{0}=\varphi
\end{aligned}
$$

Received by the editors June 5, 1992 and, in revised, July 12, 1993.

1991 Mathematics Subject Classification. Primary 47D06, 47D09.

Key words and phrases. Semigroup of operators, cosine functions of operators, compact operators.

This work was supported in part by FONDECYT Project 91-0471 and by DICYT Project 049133HM. 
is the compactness of the operator $\mathscr{T}$ defined on the continuous functions by

$$
\mathscr{T}(x(\cdot))(t)=T(t) x_{0}+\int_{0}^{t} T(t-s) F(s, x(s)) d s
$$

in the first case and by

$$
\mathscr{T}(x(\cdot))(t)=T(t) \varphi(0)+\int_{0}^{t} T(t-s) F\left(s, x_{s}\right) d s
$$

in the second one. In [5] and [6] the compactness of $\mathscr{T}$ was obtained under a compactness condition of the product $T(\cdot) F(\cdot)$. This leads us to study some weaker conditions to obtain the same result. The aim of this note is to show that if the set $\{T(\cdot) x:\|x\| \leq 1\}$ is relatively compact in the space $L^{p}([0, a] ; X)$, $1 \leq p<\infty$ and $a>0$, then $T$ is a compact semigroup.

We will represent by $L^{p}([0, a] ; X)$ the space of $p$-integrable functions from $[0, a]$ into $X, a>0$, in the Bochner's sense with respect to the Lebesgue measure, which will be designated by $m$. Furthermore, we will reserve the symbol $\alpha$ to denote the measure of noncompactness in the sense of Kuratowski. We refer to Deimling [1] for the properties of the function $\alpha$.

In the sequel we consider some fixed constants $1 \leq p<\infty$ and $a>0$. Let $d$ be a metric associated with the convergence in $m$-measure in the space $L^{p}([0, a] ; X)$. It is well known (Marle [7]) that the distance $d(f, g)$ can be defined as follows. For each $\rho>0$ we introduce the set

$$
E_{\rho}=\{t \in[0, a]:\|f(t)-g(t)\|>\rho\} .
$$

Then

$$
d(f, g):=\inf \left\{\rho>0: m\left(E_{\rho}\right) \leq \rho\right\} .
$$

The following property of the metric $d$ will be useful for us. We will use the symbol $B_{1}$ to represent the closed unit ball of $X$.

Lemma 1. Let $u:[0, a] \rightarrow \mathscr{B}(X)$ be a strongly continuous operator-valued function. If the bounded linear operator $U: X \rightarrow L^{p}([0, a] ; X), x \rightarrow u(\cdot) x$, is compact, then for each $\varepsilon>0$ there exists a finite set $\left\{x_{1}, \ldots, x_{n}\right\}$ included in $B_{1}$ such that

$$
\min _{1 \leq i \leq n} m\left(\left\{t \in[0, a]:\left\|u(t) x-u(t) x_{i}\right\|>\varepsilon\right\}\right) \leq \varepsilon
$$

for every $x \in B_{1}$.

Proof. Since $\overline{U\left(B_{1}\right)}$ is a compact set in $L^{p}([0, a] ; X)$ (Marle [7]), the uniformity induced by the convergence in $m$-measure and the uniformity induced by the norm $\|\cdot\|_{p}$ coincide on $\overline{U\left(B_{1}\right)}$. Consequently, for each $\varepsilon>0$ we can choose elements $x_{i} \in B_{1}, i=1,2, \ldots, n$, for some $n \in \mathbf{N}$, for which the following property holds: For each $x \in B_{1}$ there exists an index $i$ such that

$$
d\left(u(\cdot) x, u(\cdot) x_{i}\right)<\varepsilon .
$$

Now, using the expression (1) we obtain the assertion.

\section{RESULTS FOR SEMIGROUPS}

In this section we denote by $T$ a strongly continuous semigroup of linear operators on the Banach space $X$. We remind the reader here that the semigroup $T$ is called compact if $T(t)$ is a compact linear operator on $X$ for every $t>0$. 
We will introduce another concept of compactness using the $X$-valued functions $t \rightarrow T(t) x, x \in X$. In order to avoid repetitions we establish a general definition.

Let $u:[0, a] \rightarrow \mathscr{B}(X)$ be a strongly continuous operator-valued function. We will say that $u$ is $p$-compact if the bounded linear operator $U: X \rightarrow$ $L^{p}([0, a] ; X), x \rightarrow u(\cdot) x$, is compact.

On the other hand, by Kolmogorov-Riesz-Weils's theorem (see Vrabie [13]) a bounded set $K \subseteq L^{p}([0, a] ; X)$ is relatively compact if and only if the following conditions hold:

$\left(c_{1}\right)$ For every $\varepsilon>0$, there exists $\delta>0$ such that

$$
\int_{0}^{a}\|\tilde{f}(t+s)-f(t)\|^{p} d m(t) \leq \varepsilon^{p}
$$

for every $f \in K$ and every $s \in \mathbf{R}$ with $|s| \leq \delta$, where $\tilde{f}$ denotes the extension of $f$ defined as $\tilde{f}(t)=0$, when $t \notin[0, a]$.

$\left(c_{2}\right)$ For every measurable subset $E$ of $[0, a]$, the set $\left\{\int_{E} f d m: f \in K\right\}$ is relatively compact in $X$.

In the sequel we will compare the $p$-compactness of $u$ with the compactness of the operators $u(t)$, for each $t>0$. The following two examples show that these two concepts are not related.

Example 1. In this example we will exhibit a strongly continuous function $u:[0,1] \rightarrow \mathscr{B}\left(c_{0}\right)$ which is $p$-compact for every $1 \leq p<\infty$, but the linear operators $u(t), 0<t \leq 1$, are not compact. We define a sequence of continuous functions $f_{n}:[0,1] \rightarrow \mathbf{R}$ as follows: Let $n=2^{k}+i, k \in \mathbf{N}_{0}, i=$ $0,1,2, \ldots, 2^{k}-1$, and define $f_{n}$ to be linear on the intervals $\left[(i-1) / 2^{k}, i / 2^{k}\right]$ when $i \geq 1$ and $\left[(i+1) / 2^{k},(i+2) / 2^{k}\right]$ when $i \leq 2^{k}-2 ; f_{n}(t)=1$ for $t \in\left[i / 2^{k},(i+1) / 2^{k}\right]$ and $f_{n}(t)=0$ otherwise. The functions $f_{n}$ are clearly continuous. Now we define $u(t): c_{0} \rightarrow c_{0}$ by

$$
u(t)\left(a_{n}\right)_{n}=\left(f_{n}(t) a_{n}\right)_{n} .
$$

It is easy to see that $u(t) \in \mathscr{B}\left(c_{0}\right)$ and $u:[0,1] \rightarrow \mathscr{B}\left(c_{0}\right)$ is strongly continuous. Furthermore, from the Kolmogorov-Riesz-Weil's theorem it follows that $u$ is $p$-compact for every $1 \leq p<\infty$. However, $u(t)$ is not a compact operator since there exists an increasing sequence of natural numbers $\left(n_{j}\right)_{j}$ such that $f_{n_{j}}(t)=1$ for all $j \in \mathbf{N}$. Hence, the set $\left\{u(t) e_{n_{j}}: j \in \mathbf{N}\right\}=\left\{e_{n_{j}}: j \in \mathbf{N}\right\}$, where $e_{n}$ denotes the unitary vector $e_{n}:=\left(\delta_{n, k}\right)_{k}$, is not relatively compact in $c_{0}$.

Example 2. In this example we will construct a strongly continuous function $u:[0,1] \rightarrow \mathscr{B}(X)$ for $X=l^{1}$ such that $u(t)$ is a compact linear operator for every $t \geq 0$ but $u$ is not 1-compact. Let $\left(f_{n}\right)_{n},\left(g_{n}\right)_{n}$ be two sequences of continuous functions from $[0,1]$ into $\mathbf{R}$ which satisfy the following conditions:

(a) There exists a constant $N_{0}>0$ such that

$$
N_{0} \leq \sum_{n=1}^{\infty}\left|f_{n}(t)\right| \text { for all } 0 \leq t \leq 1 .
$$

(b) $\sum_{i=n}^{\infty}\left|f_{i}(t)\right| \rightarrow 0$, as $n \rightarrow \infty$, uniformly for $0 \leq t \leq 1$.

(c) The set $\left\{g_{n}: n \in \mathbf{N}\right\}$ is uniformly bounded and not relatively compact in the space $L^{1}([0,1] ; \mathbf{R})$. 
Let $u(t): l^{1} \rightarrow l^{1}, 0 \leq t \leq 1$, be the operator defined by $u(t)\left(a_{j}\right)_{j}=\left(b_{i}\right)_{i}$, where

$$
b_{i}:=\sum_{j=1}^{\infty} f_{i}(t) g_{j}(t) a_{j}, \quad i \in \mathbf{N} .
$$

It is well known that each operator $u(t)$ is linear, bounded, and compact (see Taylor [10, Example 5.5-3]). Furthermore, an elementary calculation using the properties of functions $f_{n}$ and $g_{n}$ shows that $u(\cdot) x$ is continuous for each $x \in l^{1}$. Nevertheless, the set $\left\{u(\cdot) x:\|x\|_{1} \leq 1\right\}$ is not relatively compact in $L^{1}\left([0,1] ; l^{1}\right)$. In fact, if $e_{n}$ denotes the unitary vector $e_{n}=\left(\delta_{i, n}\right)_{i}$ in $l^{1}$ and $N, N_{1}$ are positive constants such that

$$
\left|g_{n}(t)\right| \leq N \quad \text { for every } n \in \mathbf{N} \text { and } 0 \leq t \leq 1
$$

and

$$
\sum_{n=1}^{\infty}\left|f_{n}(t)\right| \leq N_{1} \quad \text { for every } 0 \leq t \leq 1,
$$

then, for each $h>0$, we can do the following estimations:

$$
\begin{aligned}
\int_{0}^{1-h} & \left\|u(t+h) e_{n}-u(t) e_{n}\right\|_{1} d t \\
= & \int_{0}^{1-h}\left(\sum_{i=1}^{\infty}\left|f_{i}(t+h) g_{n}(t+h)-f_{i}(t) g_{n}(t)\right|\right) d t \\
\geq & \int_{0}^{1-h}\left(\sum_{i=1}^{\infty}\left|f_{i}(t)\right|\left|g_{n}(t+h)-g_{n}(t)\right|\right) d t \\
& -\int_{0}^{1-h}\left(\sum_{i=1}^{\infty}\left|f_{i}(t+h)-f_{i}(t)\right|\left|g_{n}(t+h)\right|\right) d t \\
\geq & N_{0} \int_{0}^{1-h}\left|g_{n}(t+h)-g_{n}(t)\right| d t \\
& -N \int_{0}^{1-h}\left(\sum_{i=1}^{\infty}\left|f_{i}(t+h)-f_{i}(t)\right|\right) d t
\end{aligned}
$$

Condition (b) and the continuity of the functions $f_{i}, i \in \mathbf{N}$, imply that the second term on the right side in the above expression converges to zero as $h \rightarrow 0$ but that the first term does not converge to zero as $h \rightarrow 0$, uniformly in $n \in \mathbf{N}$, since the set $\left\{g_{n}: n \in \mathbf{N}\right\}$ is not relatively compact in $L^{1}([0,1] ; \mathbf{R})$. Consequently, the set $\left\{u(\cdot) e_{n}: n \in \mathbf{N}\right\}$ does not satisfy condition $\left(c_{1}\right)$ of Kolmogorov-Riesz-Weil's theorem, which completes the proof.

Now we will establish our main result.

Theorem 1. A strongly continuous semigroup $T$ is compact if and only if it is p-compact.

Proof. Let us assume that $T$ is a compact semigroup. This means that $T(t)$ is a compact operator for every $t>0$. We will prove that the set $K:=\{T(\cdot) x: x \in$ $\left.B_{1}\right\}$ is relatively compact in $L^{p}([0, a] ; X)$. Since $K$ is a bounded set, it is sufficient to show that $K$ satisfies conditions $\left(c_{1}\right)$ and $\left(c_{2}\right)$ above. In fact, 
in order to prove $\left(c_{1}\right)$ we observe initially that $T$ is uniformly bounded on $[0, a]$. Consequently, we may assume that $\|T(t)\| \leq M$ for $0 \leq t \leq a$ for some constant $M \geq 1$. Hence, for every pair of constants $s, \varepsilon^{\prime}>0$ sufficiently small we may write

$$
\begin{aligned}
\int_{0}^{a}\|\tilde{T}(t+s) x-T(t) x\|^{p} d t \\
=\quad \int_{0}^{\varepsilon^{\prime}}\|T(t+s) x-T(t) x\|^{p} d t \\
\quad+\int_{\varepsilon^{\prime}}^{a-s}\|T(t+s) x-T(t) x\|^{p} d t+\int_{a-s}^{a}\|T(t) x\|^{p} d t \\
\leq 2^{p} M^{p}\|x\|^{p} \varepsilon^{\prime}+s M^{p}\|x\|^{p} \\
\quad+\int_{0}^{a-s-\varepsilon^{\prime}}\left\|T(t+s) T\left(\varepsilon^{\prime}\right) x-T(t) T\left(\varepsilon^{\prime}\right) x\right\|^{p} d t .
\end{aligned}
$$

Furthermore, since $T\left(\varepsilon^{\prime}\right) B_{1}$ is a relatively compact set, there exists a constant $\delta_{\varepsilon^{\prime}}>0$ such that

$$
\|T(t+s) y-T(t) y\| \leq \varepsilon^{\prime}
$$

for all $t \in[0, a], y \in T\left(\varepsilon^{\prime}\right)\left(B_{1}\right)$, and $0<s<\delta_{\varepsilon^{\prime}}$. Substituting (4) into the right-hand side of the above inequality we deduce that for every $\varepsilon>0$ there exists $\delta>0\left(\delta=\delta_{\varepsilon^{\prime}}\right.$ for $\varepsilon^{\prime}$ appropriate) such that

$$
\int_{0}^{a}\|\tilde{T}(t+s) x-T(t) x\|^{p} d t \leq \varepsilon
$$

for every $x \in B_{1}$ and $0 \leq s \leq \delta$.

Since the proof for $s<0$ is similar we have completed the demonstration of our first assertion.

On the other hand, as $T(\cdot)$ is a strongly continuous map, Corollary 2.3 in Weis [14] implies that for every measurable set $E \subseteq[a, b]$ the operator $T_{E}$ defined by

$$
T_{E}(t) x:=\int_{E} T(t) x d m(t), \quad x \in X,
$$

is compact. Consequently the set $\left\{\int_{E} T(t) x d m(t): x \in B_{1}\right\}$ is relatively compact in $X$. This completes the proof.

Let us assume now that $T$ is $p$-compact. Since for every $w \in \mathbf{R}$ the multiplication operator

$$
M_{w}: L^{p}[(0, a] ; X) \rightarrow L^{p}([0, a] ; X), \quad f \rightarrow e^{w t} f(t),
$$

is bounded, the semigroup $e^{w t} T(t)$ is also $p$-compact. Thus, choosing $w$ appropriately we may assume that $T$ is uniformly bounded on $[0, \infty)$. Furthermore, the last property together with Lemma 1.5.1 in Pazy [9] implies that we also may suppose that $\|T(t)\| \leq 1$ for all $t \geq 0$.

On the other hand, using Lemma 1 we conclude that for every $\varepsilon>0$ there exist $k \in \mathbf{N}$ and $x_{i} \in B_{1}, i=1,2, \ldots, k$, for which the following property is satisfied. For each $x \in B_{1}$, there is $i \in\{1,2, \ldots, k\}$ such that

$$
m\left(\left\{t \in[0, a]:\left\|T(t) x-T(t) x_{i}\right\|>\varepsilon\right\} \leq \varepsilon .\right.
$$


From this property we can infer that the set of all $t$ such that

$$
\left\|T(t) x-T(t) x_{i}\right\|>\varepsilon
$$

for every $i=1,2, \ldots, k$ is included in the interval $[0, \varepsilon]$. In fact, since $\|T(t)\| \leq 1$ for all $t \geq 0$, if $\left\|T\left(t_{0}\right) x-T\left(t_{0}\right) x_{i}\right\|>\varepsilon$ for some $t_{0}>0$, then $\left\|T(t) x-T(t) x_{i}\right\|>\varepsilon$ for every $t \in\left[0, t_{0}\right]$.

Additionally, if we suppose that $\alpha\left(T(t) B_{1}\right)>0$ for some $t>0$, then there exists $n_{0} \in \mathbf{N}$ such that $\alpha\left(T(t) B_{1}\right)>1 / n$ for all $n \geq n_{0}$. From the definition of the noncompactness measure $\alpha$ it follows that $T(t) B_{1}$. is not included in $\bigcup_{j=1}^{k} B_{1 / n}\left[T(t) x_{j}\right]$, where we have denoted by $B_{r}[x]$ the closed unit ball centered at $x$ with radius $r$. Therefore, there is an element $\bar{x} \in B_{1}$ which satisfies the condition

$$
\left\|T(t) \bar{x}-T(t) x_{j}\right\|>1 / n
$$

for every $j=1,2, \ldots, k$. Now, using the preceding property, we obtain that $t \leq 1 / n$, which, in turn, implies that $t=0$. Thus, from the properties of $\alpha$ we infer that $T(t)\left(B_{1}\right)$ is relatively compact for every $t>0$.

Next we will establish a similar result for the operator-valued function $u(t)=$ $(T(t)-I)^{n}$ for $n \in \mathbf{N}$. We will denote by $A$ the infinitesimal generator of the semigroup $T$.

Lemma 2. Let $F:[0, a] \rightarrow \mathscr{B}(X)$ be a strongly continuous function such that the operator

$$
G(t):=\int_{0}^{t} F(s) d s
$$

is compact for every $t \geq 0$. Let $f:[0, a] \rightarrow \mathbf{C}$ be a continuous function. Then the operator

$$
G_{f}(t):=\int_{0}^{t} f(s) F(s) d s
$$

is also compact for all $t \geq 0$.

Proof. Let us denote by $\mathscr{K}(X)$ the closed ideal of compact operators in $\mathscr{B}(X)$. Since $f$ is uniformly continuous, there exists a sequence $\left(\varphi_{n}\right)_{n}$ of step functions which converges to $f$ uniformly. It is clear that the operator $G_{\varphi_{n}}(t) \in \mathscr{K}(X)$ for each $t \geq 0$ and $n \in \mathbf{N}$. Since the sequence $\left(G_{\varphi_{n}}(t)\right)_{n}$ converges to $G_{f}(t)$ in the uniform topology of operators, $G_{f}(t) \in \mathscr{K}(X)$.

Theorem 2. The operator $(T(t)-I)^{n}$ is compact for each $0 \leq t \leq a$ and if only if the function $u$ is $p$-compact.

Proof. If $u(t)$ is compact for $0 \leq t \leq a$, then in [3] it has been proved that $A$ is bounded and $A^{n}$ is compact. Consequently, the function $u(\cdot)$ is continuous for the uniform topology of operators, which easily implies that the set of functions $\{u(\cdot) x:\|x\| \leq 1\}$ satisfies conditions $\left(c_{1}\right)$ and $\left(c_{2}\right)$ of Kolomorov-Riesz-Weil's theorem.

Conversely, if the operator-valued function $u(\cdot)$ is $p$-compact for some $a>$ 0 , then the function $u:[0, b] \rightarrow \mathscr{B}(X)$ is $p$-compact for every $b>0$. In fact, from the expression

$$
(T(2 t)-I)^{n}=(T(t)-I)^{n}(T(t)+I)^{n}
$$


and the uniform boundedness of $(T(t)+I)^{n}$ in $[0,2 a]$ we conclude that the set $\{u(\cdot) x:\|x\| \leq 1\}$ is relatively compact in $L^{p}([0,2 a] ; X)$. Now, an easy induction proves the assertion.

Let $F_{n}(\lambda)$ be the Laplace transform of the function $u(\cdot)$. Since

$$
F_{n}(\lambda)=\lim _{a \rightarrow \infty} \int_{0}^{a} e^{-\lambda t} u(t) d t
$$

and the convergence is in the uniform sense, by Lemma 2 we obtain that $F_{n}(\lambda)$ is a compact operator for $\lambda \in \mathbf{R}$ large enough. Thus, Theorem 2.4 in Henríquez [3] implies that $(T(t)-I)^{n}$ is compact for all $t \geq 0$.

\section{RESULTS FOR COSINE FUNCTIONS}

Let us consider now a strongly continuous cosine function of linear operator $C$ defined on $X$. Let $S$ be the sine function associated to $C$, which is defined by

$$
S(t) x:=\int_{0}^{t} C(s) x d s, \quad x \in X, t \in \mathbf{R} .
$$

For the general properties of cosine function of operators we refer to [2] and [11].

Theorem 3. The cosine function $C$ is p-compact if and only if $X$ is a finitedimensional space.

Proof. If $X$ is a finite-dimensional space, then the map $C(\cdot)$ is continuous for the norm of operators and each $C(t)$ is a compact operator. From AscoliArzelá's Theorem is easy to see that the set of functions $\left\{C(t) x: x \in B_{1}\right\}$ is relatively compact in the space of continuous functions $C([0, a] ; X)$. Since $C([0, a] ; X)$ is continuously included in $L^{p}([0, a] ; X)$, it follows that $C$ is p-compact.

Conversely, if $C$ is $p$-compact restricted to the interval $[0, a]$, then $C$ is also $p$-compact on the interval $[0, a / 2]$. Further, as $C$ is uniformly bounded on $[0, a / 2]$, a simple calculus shows that the map $t \rightarrow C^{2}(t)$ is $p$-compact on $[0, a / 2]$. These properties and the identity [2]

$$
C(2 t)=2 C^{2}(t)-I, \quad t \in \mathbf{R},
$$

allow us to conclude that the constant operator-valued map $[0, a / 2] \rightarrow \mathscr{B}(X)$, $t \rightarrow I$, is $p$-compact. From the definition of $p$-compactness and condition $\left(c_{2}\right)$ we obtain easily that $I$ is compact, which in turn implies that $X$ has finite dimension.

Next we establish the analogue of Theorem 1 for the sine function.

Theorem 4. The sine function $S$ is p-compact if and only if $S(t)$ is a compact operator for every $t \in \mathbf{R}$.

Proof. From expression (6) it follows easily that the operator-valued function $S(\cdot)$ is continuous for the norm of operators. Consequently, if $S(t)$ is a compact operator for each $t \in \mathbf{R}$, then Ascoli-Arzelá's Theorem implies that the set $\left\{S(t) x: x \in B_{1}\right\}$ is relatively compact in the space of continuous functions and, proceeding as in the Theorem 3, we conclude that $S$ is $p$-compact. 
Conversely, if $S$ is a $p$-compact map on an interval $[0, a]$, using the fact that $S(\cdot)$ is continuous for the operator norm and the condition $\left(c_{2}\right)$ we obtain that the operator

$$
\frac{1}{h} \int_{t}^{t+h} S(s) d s, \quad 0 \leq t<a, h>0,
$$

is compact and converges uniformly to $S(t)$, as $h \rightarrow 0^{+}$. Consequently, $S(t)$ is compact for $0 \leq t \leq a$. From the properties of cosine functions [12] we obtain that $S(t)$ is compact for all $t \in \mathbf{R}$.

We conclude this note with the analogue of Theorem 2 for cosine functions. Let $u(t)=(C(t)-I)^{n}, n \in \mathbf{N}$.

Theorem 5. The operator $(C(t)-I)^{n}$ is compact for all $t \in \mathbf{R}$ if and only if the function $u(\cdot)$ is p-compact.

We omit the proof since the argument is very similar to that employed in the demonstration of Theorem 2 . We only need to use the expression

$$
(C(2 t)-I)^{n}=2^{n}(C(t)-I)^{n}(C(t)+I)^{n}
$$

instead of (5) and the results established in [4] instead of those in [3].

\section{REFERENCES}

1. K. Deimling, Nonlinear functional analysis, Springer-Verlag, Berlin, 1985.

2. H. O. Fattorini, Second order linear differential equations in Banach spaces, North-Holland, Amsterdam, 1985.

3. H. R. Henríquez, Uma propriedade compacidade para familias cosseno de operadores, Proc. $14^{\circ}$ Colóquio Brasileiro de Matemática, Instituto de Matemática Pura e Aplicada, Rio de Janeiro, Brasil, 1983, pp. 71-80.

4. __ Una nota sobre la compacidad de funciones coseno de operadores, Revista Proyecciones 6 (1987), 35-46.

5. __ Periodic solutions of quasi-linear partial functional differential equations with unbounded delay, Funkcial. Ekvac. (to appear).

6. J. H. Lightbourne, Periodic solutions and perturbed semigroups of linear operators, Nonlinear System and Applications, Academic Press, New York, 1977, pp. 591-602.

7. C. M. Marle, Mesures et probabilités, Hermann, Paris, 1974.

8. R. Nagel, One parameter semigroups of positive operators, Lecture Notes in Math., vol. 1184, Springer-Verlag, Berlin, 1986.

9. A. Pazy, Semigroups of linear operators and applications to partial differential equations, Springer-Verlag, New York, 1983.

10. A. E. Taylor, Introduction to functional analysis, Wiley, New York, 1958.

11. C. C. Travis and G. F. Webb, Second order differential equations in Banach space, Proc. Internat. Sympos. on Nonlinear Equations in Abstract Spaces, Academic Press, New York, 1987, pp. 331-361.

12. Compactness, regularity and uniform continuity properties of strongly continuous cosine families, Houston J. Math. 3 (1977), 555-567.

13. I. I. Vrabie, Compactness methods for nonlinear evolutions, Longman Sci. Tech., Essex, 1987.

14. L. W. Weis, $A$ generalization of the Vidav-Jorgens perturbation theorem for semigroups and its application to transport theory, J. Math. Anal. Appl. 129 (1988), 6-23.

Departamento de Matemática, Universidad de Santiago, Casilla 307, Correo 2, Santiago, Chile 